\title{
Molecular mechanism of miR-181b in heart disease due to pregnancy-induced hypertension syndrome
}

\author{
ZHENG GAO $^{1}$, LI WANG ${ }^{2}$, JINYUN WANG ${ }^{1}$, FENGYONG YANG $^{3}$ and JIN QU ${ }^{4}$ \\ ${ }^{1}$ Department of Obstetrics and Gynecology, Laiwu City People's Hospital, \\ Laiwu, Shandong 271100; ${ }^{2}$ Department of Gynecology, Affiliated Hospital of \\ Shandong University of Traditional Chinese Medicine, Jinan, Shandong 250011; \\ ${ }^{3}$ Intensive Care Unit (ICU), Laiwu City People's Hospital, Laiwu, Shandong 271100; \\ ${ }^{4}$ Ministry of Painless Endoscopy, Jining No. 1 People's Hospital, Jining, Shandong 272000, P.R. China
}

Received July 21, 2015; Accepted January 6, 2017

DOI: $10.3892 / \mathrm{etm} .2017 .4882$

\begin{abstract}
The present study aimed to investigate the molecular mechanisms of microRNA (miR)-181b in heart disease due to hypertensive disorders complicating pregnancy (HDCP) through regulating the expression of metallopeptidase inhibitor 3 (TIMP3). miR-181b expression was detected by reverse transcription-quantitative polymerase chain reaction in peripheral blood samples from patients with HDCP. These samples were analyzed for clinical pathological characteristics. The primary cardiomyocytes of rats were cultured in hypoxic conditions for $24 \mathrm{~h}$, in which miR-181b expression was detected at different time points. The expression of TIMP3 was assessed in normal rat cardiomyocytes following transfection with miR-181b mimics by western blot analysis. The TIMP3 protein was also detected in cardiomyocytes following transfection with TIMP3 short interfering-RNA. The apoptosis rate of transfected cardiomyocytes was detected by flow cytometry following $24 \mathrm{~h}$ of culture in a hypoxic environment. Luciferase assay was applied to validate whether miR-181b binds to the 3' untranslated region of TIMP3 mRNA. miR-181b expression was significantly downregulated in the peripheral blood of patients with HDCP and the miR-181b expression was negatively associated with the grades of hypertension $(\mathrm{P}<0.05)$. The results of luciferase assay indicated that miR-181b directly targets TIMP3. The apoptosis rates of rat cardiomyocytes in the group transfected with miR-181b or TIMP3 siRNA was significantly lower than the normal control group $(\mathrm{P}<0.05)$. miR-181b may inhibit apoptosis of cardiomyocytes to protect
\end{abstract}

Correspondence to: Dr Jin Qu, Ministry of Painless Endoscopy, Jining No. 1 People's Hospital, 6 Jiankang Road, Jining, Shandong 272000, P.R. China

E-mail: zgzz007@163.com

Key words: pregnancy-induced hypertension syndrome, pregnancy induced hypertension, metallopeptidase inhibitor 3 myocardial cells through directly targeting TIMP3 genes, which serve important roles in HDCP.

\section{Introduction}

Hypertensive disorder complicating pregnancy (HDCP) is a rare disease in pregnant women, which is a primary cause of mortality for pregnant women and perinatal children, although its pathogenesis remains unclear (1-3). The incidence of HDCP is increasing, which in turn increases the risk to the health of pregnant women and perinatal children (4). In China, the morbidity is $\sim 10 \%$ and the incidence rate is $7-12 \%$ in other countries $(5,6)$. Heart disease is one of the diseases induced by HDCP, the pathological characteristics of which include myocardial injury and may lead to cardiac failure (7). HDCP may increase the preload of the heart and induce cardiomyocyte hypertrophy and eclampsia, which causes damage to myocardial cells and potentially injures the developing fetus $(8,9)$. The mechanisms remain unclear and therefore, it is valuable for early diagnosis and treatment to investigate the molecular mechanisms of myocardial injury induced by HDCP.

miRNA's are a class of post-transcriptional regulators 18-22 nucleotides in length, which serve important roles in the processes of myocardial cell development, apoptosis and injury (10-12). A previous study demonstrated that the miR-181 family served important functions during the progression of cancer, hypertension, myocardial fibrosis and other diseases (13). Banzet et al (14) revealed that miR-181b expression increased when volume became overloaded in heart. Jiang et al (15) demonstrated that miR-181b expression was significantly decreased in cardiac fibroblasts when treated with angiotensin 2 although its mechanism remains unclear. In the current study, metallopeptidase inhibitor 3 (TIMP3) was predicted to be a candidate target gene for miR-181b using bioinformatics methods. TIMP3 is an important inhibitor of matrix metalloproteinases (MMP), which serve a role in myocardial injury and ventricular remodeling (15). The present study aimed to investigate miR-181b expression and the expression of associated target gene, TIMP3, in myocardial injury, which may help to elucidate the clinical values of miR-181b in heart disease associated with HDCP. 


\section{Materials and methods}

Sample collection. The current study consisted of 46 cesarean section puerperas with HDCP and 30 normal delivery puerperas. The peripheral blood was collected from patients from February 2013 to November 2014 in Laiwu City People's Hospital (Laiwu, China; n=14), Affiliated Hospital of Shandong University of Traditional Chinese Medicine (Jinan, China; $\mathrm{n}=17$ ) and Jining No. 1 People's Hospital (Jining, China; $\mathrm{n}=15$ ). A single peripheral blood sample was collected from each patient or control $2 \mathrm{~h}$ before delivery. According to the blood pressure level and clinical classification standard in HDCP patients (16), the 46 patients are classified into grades as follows: 28 patients in grade I, 13 in grade II and 5 in grade III. The average age of patients in the grade III group was 28.6 years and the median age was 28 years (23.5-35 years). The average age of patients in the normal control group was 27.5 years old, and the median was 26 years old. The details of patients are presented in Table I. Prior written and informed consent were obtained from every patient and the study was approved by the Ethics Review Board of Laiwu City People's Hospital, Affiliated Hospital of Shandong University of Traditional Chinese Medicine and Jining No. 1 People's Hospital.

Primary culture of myocardial cells. A total of 10 female Sprague Dawley rats aged 5-6 weeks, and weighing 150-200 g were purchased from Vital River Laboratory Animal Technology Co., Ltd. (Beijing, China) and were housed at $25^{\circ} \mathrm{C}$ with $30 \%$ humidity and a $12 \mathrm{~h}$ light/dark cycle, and with free access to water and food. After experiment, the animals were sacrificed by cervical dislocation. The cardiac apex was removed and washed with normal saline twice from 3-day-old newborn Sprague Dawley rats by cutting along the sternum midlines of the chest wall. The tissue was cut into sections $\sim 1 \mathrm{~mm}^{3}$ using ophthalmic scissors, then washed twice in phosphate buffered saline (PBS) without calcium and magnesium ions. The tissue pieces were moved into $10 \mathrm{ml}$ centrifuge tubes for digestion by trypsin and type II collagenase. The myocardial cells were collected by centrifugation at $500 \mathrm{x}$ g for $5 \mathrm{~min}$ at room temperature. The collected cells were cultured for 2 days at $37^{\circ} \mathrm{C}$ in an atmosphere containing $5 \% \mathrm{CO}_{2}$ with High glucose-Dulbecco's modified Eagle's medium (DMEM) containing $10 \%$ fetal bovine serum (FBS; BD Biosciences, Franklin Lakes, NJ, USA).

RNA extraction and reverse transcription from peripheral blood samples. The supernatant was collected following centrifugation at $2,000 \mathrm{x}$ g for $10 \mathrm{~min}$ at room temperature from a $5 \mathrm{ml}$ anticoagulated peripheral blood sample from each patient. A total of $250 \mu \mathrm{l}$ serum was mixed with $750 \mathrm{ml}$ TRIzol (Invitrogen; Thermo Fisher Scientific, Inc., Waltham, MA, USA) to extract total RNA based on the phenol-chloroform method (17). The miR cDNA was synthesized from total RNA by reverse transcription with an miRNA cDNA kit (Takara Biotechnology, Co., Ltd., Dalian, China). The reverse system of miRNA (miScript II RT kit; Qiagen, AB, Sollentuna, Sweden) included: $5 \mu \mathrm{l}$ miRNA template, $10 \mu \mathrm{l}$ $2 \mathrm{X}$ miRNA Reaction Buffer mix, $2 \mu \mathrm{l} 0.1 \%$ bovine serum albumin, $2 \mu \mathrm{l}$ miRNA PrimeScript RT Enzyme Mixture
Table I. Details of 46 patients with a hypertensive disorder complicating pregnancy.

\begin{tabular}{|c|c|c|c|c|}
\hline Patient & $\begin{array}{l}\text { Age, } \\
\text { years }\end{array}$ & $\begin{array}{c}\text { Systolic/ } \\
\text { diastolic } \\
\text { pressure, } \\
\text { mmHg }\end{array}$ & Grade & Hospital \\
\hline 1 & 22 & $148 / 94$ & I & 1 \\
\hline 2 & 24 & $152 / 92$ & I & 2 \\
\hline 3 & 28 & $145 / 93$ & I & 1 \\
\hline 4 & 27 & $147 / 90$ & I & 3 \\
\hline 5 & 26 & $144 / 95$ & I & 2 \\
\hline 6 & 28 & $147 / 95$ & I & 2 \\
\hline 7 & 29 & $152 / 96$ & I & 3 \\
\hline 8 & 30 & $152 / 92$ & I & 2 \\
\hline 9 & 26 & $143 / 90$ & I & 1 \\
\hline 10 & 31 & $144 / 90$ & I & 3 \\
\hline 11 & 27 & 148/91 & I & 2 \\
\hline 12 & 27 & $149 / 99$ & I & 1 \\
\hline 13 & 27 & $154 / 92$ & I & 1 \\
\hline 14 & 27 & $151 / 97$ & I & 2 \\
\hline 15 & 29 & $152 / 95$ & I & 3 \\
\hline 16 & 25 & $146 / 90$ & I & 3 \\
\hline 17 & 26 & $143 / 94$ & I & 3 \\
\hline 18 & 26 & $149 / 97$ & I & 2 \\
\hline 19 & 27 & $150 / 92$ & I & 1 \\
\hline 20 & 28 & $151 / 90$ & I & 1 \\
\hline 21 & 27 & $152 / 92$ & I & 2 \\
\hline 22 & 28 & $141 / 90$ & I & 2 \\
\hline 23 & 27 & $140 / 93$ & I & 2 \\
\hline 24 & 27 & $142 / 92$ & I & 3 \\
\hline 25 & 27 & $144 / 90$ & I & 3 \\
\hline 26 & 26 & $151 / 90$ & I & 3 \\
\hline 27 & 27 & $143 / 98$ & I & 2 \\
\hline 28 & 28 & $145 / 97$ & I & 1 \\
\hline 29 & 26 & $165 / 102$ & II & 3 \\
\hline 30 & 32 & $161 / 105$ & II & 2 \\
\hline 31 & 31 & $165 / 101$ & II & 1 \\
\hline 32 & 28 & $162 / 105$ & II & 1 \\
\hline 33 & 22 & $170 / 107$ & II & 2 \\
\hline 34 & 24 & $171 / 101$ & II & 3 \\
\hline 35 & 28 & $161 / 109$ & II & 1 \\
\hline 36 & 25 & $165 / 108$ & II & 3 \\
\hline 37 & 26 & $168 / 102$ & II & 2 \\
\hline 38 & 25 & $167 / 100$ & II & 3 \\
\hline 39 & 24 & $165 / 106$ & II & 1 \\
\hline 40 & 26 & $170 / 100$ & II & 2 \\
\hline 41 & 27 & $164 / 103$ & II & 3 \\
\hline 42 & 35 & $180 / 110$ & III & 1 \\
\hline 43 & 28 & $180 / 110$ & III & 1 \\
\hline 44 & 32 & $180 / 110$ & III & 2 \\
\hline 45 & 34 & $210 / 110$ & III & 2 \\
\hline 46 & 30 & $185 / 110$ & III & 3 \\
\hline
\end{tabular}

1, Laiwu City People's Hospital (Laiwu, China); 2, Affiliated Hospital of Shandong University of Traditional Chinese Medicine (Jinan, China); 3, Jining NO. 1 People's Hospital (Jining, China). 
(Takara Biotechnology, Co., Ltd.,) and $1 \mu 1 \mathrm{H}_{2} \mathrm{O}$. The reaction was completed at $37^{\circ} \mathrm{C}$ for 60 min with PolyA primer, then the reaction solution was added to RT RNAase Free $\mathrm{H}_{2} \mathrm{O}$ to a total volume of $50 \mu \mathrm{l}$. Quantitative detection was completed according to the manufacturer's protocol as previously described (18) (miScript SYBR-Green PCR kit; Qiagen AB). The system included the following: $10 \mu \mathrm{l}$ qRT-PCR-Mix, $0.5 \mu \mathrm{l}$ forward primers and $0.5 \mu \mathrm{l}$ reverse primers, $5 \mu \mathrm{l} \mathrm{cDNA}$

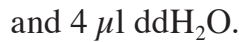

Reverse transcription-quantitative polymerase chain reaction $(R T-q P C R)$ to detect the expression of miR-181b. The miScript SYBR-Green PCR kit (Qiagen AB) was used to detect the expression of miR-181b in peripheral blood samples. U6 was used as internal reference. The primers were as follows: miR-181b forward, 5'-AACATTCATTGCTGTCGGTGGGT-3' (reverse primer was provided in the kit without sequence information); U6 forward, 5'-CTCGCTTCGGCAGCACA-3' and reverse, 5'-AACGCTTCACGAATTTGCGT-3'; and Uni-miR primers were provided in the kit without sequence information. The miRNA reaction system included $10 \mu \mathrm{l}$ RT-qPCR-Mix, $0.5 \mu \mathrm{l}$ forward primer and $0.5 \mu 1$ reverse primer, $5 \mu \mathrm{l} \mathrm{cDNA}$

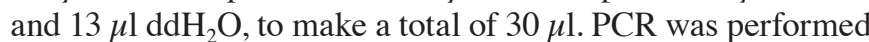
using a thermocycler (StepOne Plus; Thermo Fisher Scientific, Inc.) as follows: $95^{\circ} \mathrm{C}$ for $10 \mathrm{~min}$ followed by 40 cycles of $95^{\circ} \mathrm{C}$ for $30 \mathrm{sec}$ and $60^{\circ} \mathrm{C}$ for $30 \mathrm{sec}$. Each sample had 3 replicates and the average was used.

Detecting expression of miR-181b and TIMP3 in primary cardiomyocytes with anoxia. The primary myocardial cells were cultured in an environment of $1 \% \mathrm{O}_{2}, 5 \% \mathrm{CO}_{2}$ and $94 \% \mathrm{~N}_{2}$. Following 2, 4, 8, 12 and $24 \mathrm{~h}$, cells were collected to extract total RNA according to the phenol-chloroform extraction protocol. Following reverse transcription, the expression of miR-181b and TIMP3 was detected by RT-qPCR. The primers of TIMP3 were as follows: Forward 5'-GAAGAG AGTACCGGCATCGG-3' and reverse 5'-CCATTCTCCCCC TGCCAAAT-3'. PCR was performed ysing the StepOne Plus thermocycler as follows: $95^{\circ} \mathrm{C}$ for $10 \mathrm{~min}$ and 40 cycles of $95^{\circ} \mathrm{C}$ for $30 \mathrm{sec}, 60^{\circ} \mathrm{C}$ for $30 \mathrm{sec}$ and $72^{\circ} \mathrm{C} 30 \mathrm{sec}$, followed by $1 \mathrm{~min}$ at $72^{\circ} \mathrm{C}$ for extension. The step for detecting miR-181b was completed as previously described.

Myocardial cell transfection. The primary myocardial cells were divided into 4 groups based on transfection: Negative control, miR-181b mimic, miR-181b inhibitor, and TIMP3-short interfering RNA (siRNA). The cells were cultured in antibiotic-free $\mathrm{H}$-DMEM medium with $10 \% \mathrm{FBS}$ at $37^{\circ} \mathrm{C}$ and transfected at $70 \%$ confluence. A total of $25 \mathrm{pmol} / \mu \mathrm{l}$ negative controls and small interfering RNA and 1.25 or $1 \mu \mathrm{l}$ Lipofectamine 2000 (Invitrogen; Thermo Fisher Scientific, Inc.) was added into Eppendorf tubes containing $50 \mu \mathrm{l} \mathrm{Opti}$ Memi medium (Thermo Fisher Scientific, Inc.), respectively. The two tubes were mixed following $5 \mathrm{~min}$ of standing, followed by another $20 \mathrm{~min}$ standing at room temperature. The mixture was added to each well when the cell density reached $70-90 \%$. The H-DMEM medium with $10 \%$ FBS was replaced following $6 \mathrm{~h}$ of transfection. Following $48 \mathrm{~h}$ of transfection, at which point the interfering effect was evident, TIMP3 protein expression was detected by western blot analysis.
Western blot analysis. Following $48 \mathrm{~h}$ transfection, the primary myocardial cells were washed twice using pre-cooled PBS, then lysed with radioimmunoprecipitation assay lysis buffer (Beyotime Institute of Biotechnology, Haimen, China) with $1 \%$ phenylmethylsulfonyl fluoride (Beyotime Institute of Biotechnology) on ice for $5 \mathrm{~min}$. Mixed with 5X SDS loading buffer and incubated at $100^{\circ} \mathrm{C}$ for $10 \mathrm{~min}, 20 \mu \mathrm{g}$ of protein was loaded into SDS-PAGE at $100 \mathrm{~V}$ constant voltage. The proteins were transferred to a polyvinylidene fluoride membrane at $300 \mathrm{~mA}$ constant current for $1 \mathrm{~h}$. Following blocking for $1 \mathrm{~h}$ using 5\% skim milk at room temperature, the primary antibodies were added to incubate at $4^{\circ} \mathrm{C}$ overnight. The primary antibodies used were mouse anti-mouse GAPDH antibody (ab8245; 1:2,000) and rabbit anti-mouse TIMP3 (ab39184; 1:800). The secondary antibody was horseradish peroxidase-conjugated (HRP) goat anti-rabbit immunoglobulin G (ab6721; 1:10,000) and HRP goat anti-mouse immunoglobulin G (ab6789; 1:10,000). All antibodies were purchased from Abcam (Cambridge, MA, USA). The membrane was developed and exposed by ECL of the BeyoECL plus kit (Beyotime Institute of Biotechnology). Quantity One software version 4.6.2 (Bio-Rad Laboratories, Inc., Hercules, CA, USA) was used to carry out the quantitative analysis. The experiment was performed in triplicate.

Annexin V-fluorescein isothiocyanate (FITC) staining and flow cytometry analysis. To investigate the role of miR-181b in myocardial injury, the present study analyzed the apoptosis rate of the negative control group, miR-181b mimic group and TIMP3 siRNA group, all of which were cultured in hypoxia for $24 \mathrm{~h}$. Annexin V-FITC staining was performed according to the protocol of the FITC Annexin V Apoptosis Detection kit I (BD Biosciences). Briefly, cells were digested and rinsed with PBS. $1 \times 10^{6}$ cells were seeded and incubated for $15 \mathrm{~min}$ at room temperature with 5 ul PI and FITC coupling Annexin V, and BD FACSVerse flow cytometry (BD Biosciences) was subsequently used to detect the apoptosis rate of cells in each group. Positive Annexin v indicates the apoptosis cells are in early stage, while propidium iodide (PI) positive means the cells are necrotic. Annexin V and PI double positive indicates that cells are in late stage apoptosis. The analysis was performed using the BD FACSuite Software v1.0.3 provided with the flow cytometer.

Dual luciferase assay. Based on the bioinformatics prediction, the mutant 3 ' untranslated region (UTR) of TIMP3 and the wild-type 3'UTR were synthesized in vitro (Hanbio Biotechnology Co., Ltd., Shanghai, China) and cloned into the downstream region of the pMIR-REPORT luciferase vector (Hanbio Biotechnology Co., Ltd.) using HindIII and Spe1 restriction enzymes. HEK293T cells were co-transfected with miR-181b mimic and wild type TIMP3 3'UTR or the mutant 3'UTR. Following $24 \mathrm{~h}$ transfection, cells were lysed and luciferase intensity was measured using the GloMax ${ }^{\circledR}$ 20/20 luminometer (Promega Corporation, Madison, WI, USA). The intensity of Renilla was used as control, and the protocol of the Dual Luciferase Reporter Gene Assay kit (Beyotime Institute of Biotechnology) was followed.

Statistical analysis. SPSS 17.0 (SPSS Inc., Chicago, IL, USA) software was applied for statistical analysis. All data is 
presented as the mean \pm standard deviation and differences were determined by two-tailed Student's t-test. $\mathrm{P}<0.05$ was considered to represent statistically significant differences.

\section{Results}

miR-181b expression in peripheral blood samples. To investigate the roles of miR-181b in HDCP, RT-qPCR was used to detect miR-181b expression in peripheral blood samples obtained from HDCP patients and the control. As Fig. 1A demonstrates, miR-181b expression was significantly lower in peripheral blood of patients with HDCP compared with the control puerpera $(\mathrm{P}<0.05)$. miR-181b expression was increased in patients in stage II/III hypertension compared with those in stage I (Fig. 1B). For detecting the correlation of miR-181b expression with TIMP3 expression, another 15 patients with HDCP and 15 healthy individuals were selected from the aforementioned patients to estimate the expression in blood. As Fig. 1C demonstrates, TIMP3 was significantly increased in patients with HDCP, which was negatively associated with the expression of miR-181b.

Expression of miR-181b and TIMP3 in injured myocardial cells. To assess the participation of miR-181b in the processes of hypoxia-injured myocardial cells, RT-qPCR was used to detect miR-181b expression and TIMP 3 expression at different time points for hypoxia culturing. It was demonstrated that miR-181b expression was significantly decreased along with the duration of hypoxia in culturing cells $(\mathrm{P}<0.05)$. The expression of TIMP3 was significantly increased with the hypoxia time extension $(\mathrm{P}<0.05)$. miR-181b expression was negatively associated with TIMP3 expression, as presented in Fig. 2.

Expression of TIMP3 in myocardial cells. To investigate whether miR-181b has an influence on the expression of TIMP3 in myocardial cells, western blot analysis was completed, in order to detect the expression of TIMP3 protein in different groups of cells. The proteins were isolated in different groups of cells transfected with miR-181b mimics, miR-181b inhibitor and TIMP3 siRNA. It was indicated that TIMP3 protein expression was downregulated when miR-181b was upregulated (miR-181b mimic group), however the expression of TIMP3 increased when miR-181b was significantly downregulated (miR-181b inhibitor group; $\mathrm{P}<0.05$; Fig. 3A). The expression of TIMP3 significantly decreased in the TIMP3 siRNA group $(\mathrm{P}<0.05$; Fig. 3B).

The apoptosis influenced by miR-181b and TIMP3. To investigate the influence on apoptosis by miR-181b and TIMP3, flow cytome-try was applied to detect the rate of apoptotic cells using Annexin V and PI double staining. As Fig. 4 demonstrates, following culture in a hypoxic environment for $24 \mathrm{~h}$, the apoptosis rate of myocardial cells in the miR-181b mimic group was significantly lower than in the negative control group $(\mathrm{P}<0.05)$. The rate of apoptosis also significantly decreased in the TIMP3 siRNA group compared with the negative control group $(\mathrm{P}<0.05)$. Combined with the bioinformatics prediction, it was indicated that miR-181b may inhibit the apoptosis of myocardial cells through regulating TIMP3 expression.
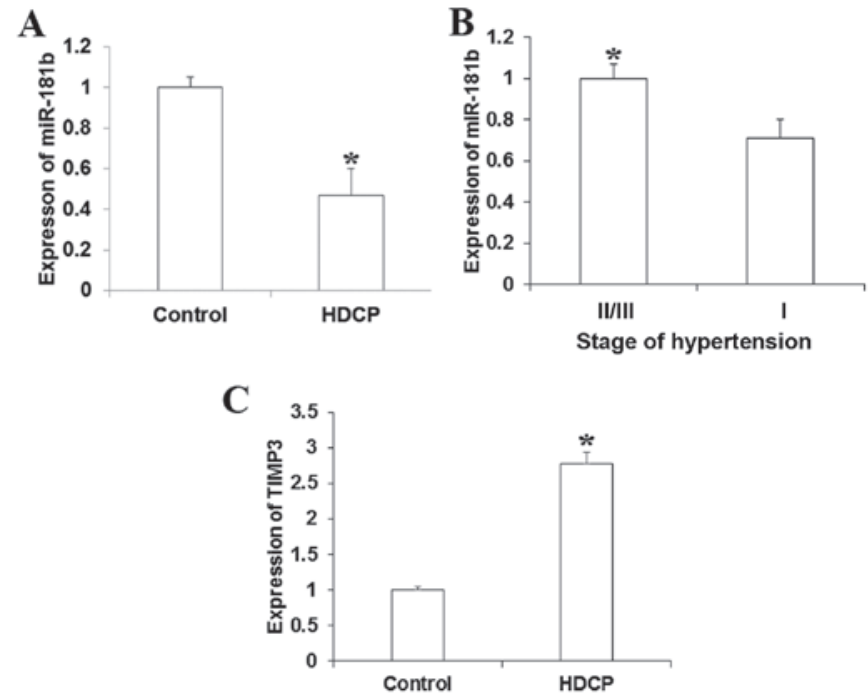

Figure 1. Expression of miR-181b in peripheral blood relative to U6 (fold) The expression of miR-181b was detected using reverse transcription-quantitative polymerase chain reaction. (A) Expression of miR-181b increased in the peripheral blood of patients with HDCP compared with normal control. (B) miR-181b expression decreased in patients in stage III and IV compared with stage II hypertension. (C) The expression of TIMP3 was significantly increased in peripheral blood samples of patients with HDCP. "P<0.05. miR, microRNA; HDCP, hypertensive disorder complicating pregnancy; TIMP3, metallopeptidase inhibitor 3 .

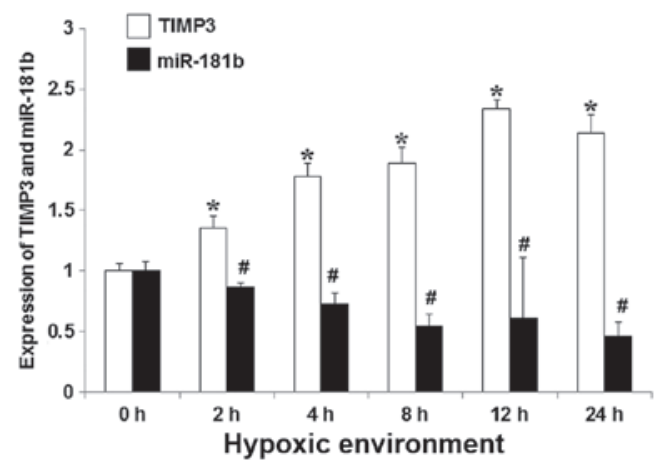

Figure 2. The correlation between miR-181b and TIMP3 expressions relative to controls at specific time points during culture in hypoxia. Assessed using reverse transcription-quantitative polymerase chain reaction. miR-181b expression was significantly decreased in a time-dependent manner in association with hypoxia. TIMP3 expression was significantly increased with the duration of hypoxia. ${ }^{*} \mathrm{P}<0.05$ for miR-181b expression vs. $0 \mathrm{~h},{ }^{\#} \mathrm{P}<0.05$ for TIMP3 expression vs. $0 \mathrm{~h}$. miR, microRNA; TIMP3, metallopeptidase inhibitor 3 .

miR-181b directly targets TIMP 3 expression. To determine whether TIMP3 was directly regulated by miR-181b, the luciferase intensity was detected using a dual luciferase assay. As presented in Fig. 5A, the binding sites of miR-181b seed region on the TIMP3 3'UTR were mutated in the in vitro synthesized sequence. The present study identified that the luciferase intensity was significantly decreased when co-transfected with miR-181b mimics and TIMP3 3'UTR luciferase vector compared with the negative control $(\mathrm{P}<0.05)$, while no significant difference between the negative control and the mutant TIMP3 3'UTR was observed (P>0.05; Fig. 5B). The results of the current study indicate that miR-181b may directly bind to the 'seed region' in 3'UTR of TIMP3 mRNA to regulate TIMP3 expression. 
A
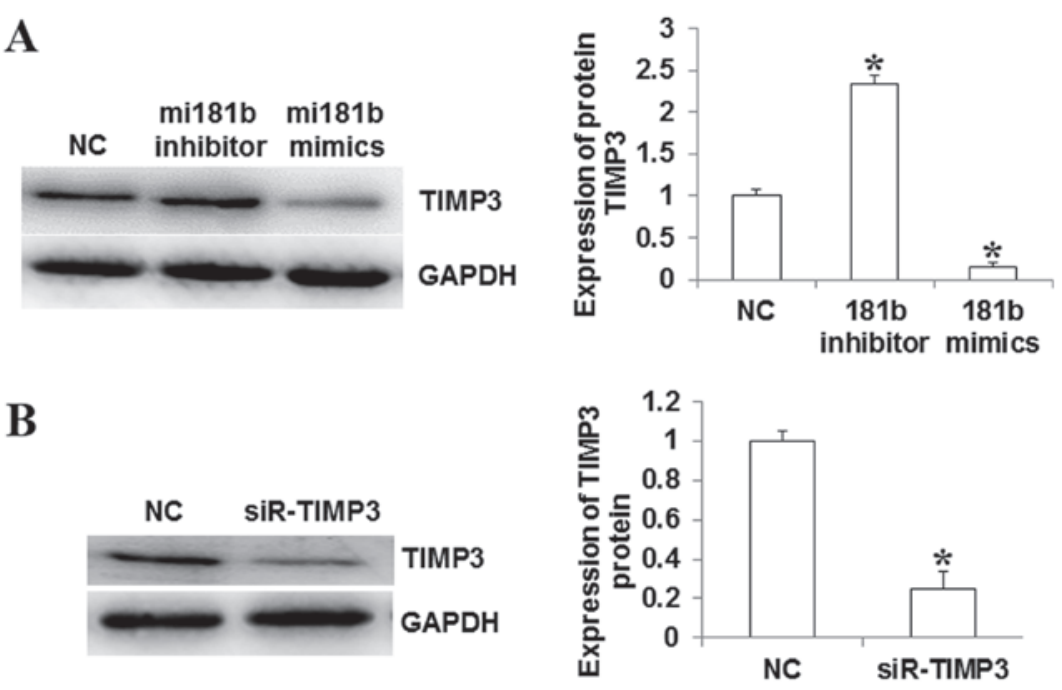

Figure 3. Expression of TIMP3 in myocardial cells, detected by western blot analysis. (A) TIMP3 expression relative to U6 increased in myocardial cells following transfection with miR-181b inhibitor and decreased following transfection with miR-181b mimic, compared with the control group. (B) TIMP3 expression decreased in myocardial cells following transfection with siR-TIMP3 compared with control cells. "P $<0.05$ vs. NC. TIMP3, metallopeptidase inhibitor 3; miR, microRNA; NC, normal control; GAPDH, glyceraldehyde 3-phosphate dehydrogenase; siR-TIMP, TIMP3 short interfering RNA.
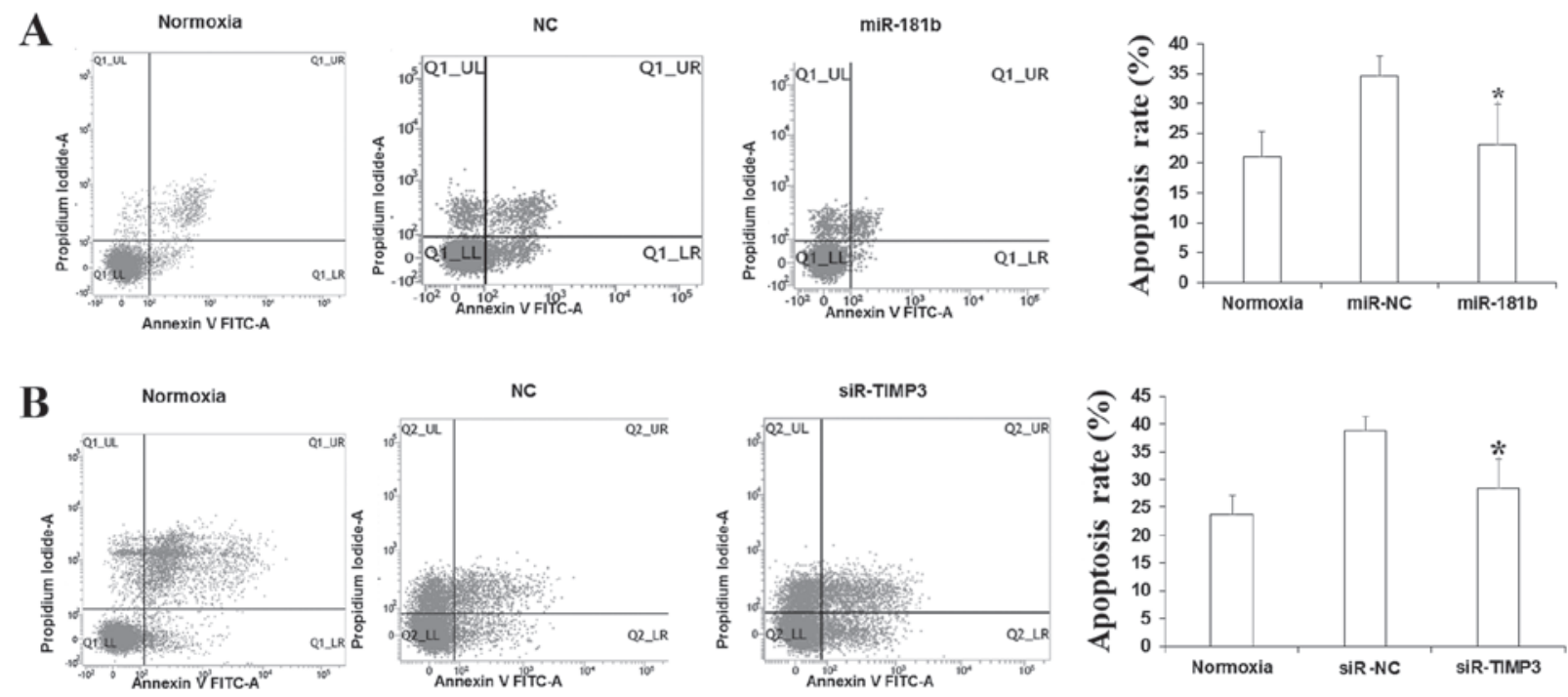

Figure 4. miR-181b inhibits the apoptosis of myocardial cells. (A) Rate of apoptosis in myocardial cells following transfection with miR-181b was lower than that of the NC. (B) Rate of apoptosis in myocardial cells following transfection with siR-TIMP3 was lower than that of the NC. ${ }^{*} \mathrm{P}<0.05 \mathrm{vs}$. NC. miR, microRNA; NC, normal control; siR-TIMP3, metallopeptidase inhibitor 3 short interfering RNA; FITC, fluorescein isothiocyanate.

A

\begin{tabular}{|c|c|}
\hline WT & $5^{\prime}$....GGUGAAAGACUGAGAUGAAUGUC \\
\hline & || ||| || \\
\hline Has-miR-181b & 3' UGGGUGGCUGUCGUUACUUACAA \\
\hline \multirow[t]{2}{*}{ Mutant } & 5' ....GGUGAAAAAGUGAGAGGGAGGGC... \\
\hline & I I I \\
\hline Has-miR-181b & 3' UGGGUGGCUGUCGUUACUUACAA \\
\hline
\end{tabular}

B

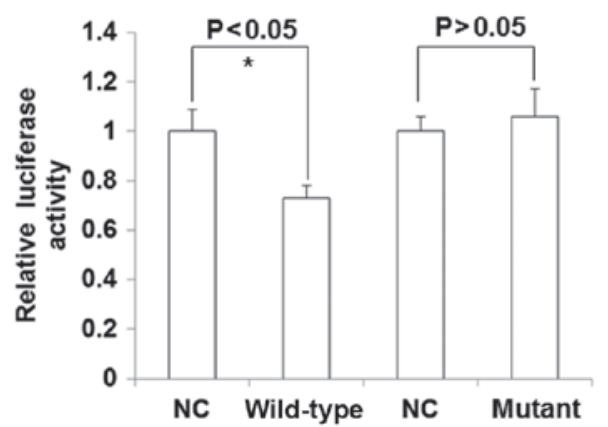

Figure 5. TIMP3 is a direct target gene of miR-181b. (A) miR-181b seed sequence and binding sites on TIMP3 3 'untranslated region (upper) and a number of base mutations. (B) Dual luciferase assay used to detect whether TIMP3 is regulated by miR-181b. Luciferase activity relative to controls. ${ }^{*} \mathrm{P}<0.05 \mathrm{vs}$. NC. TIMP3, metallopeptidase inhibitor 3; miR, microRNA; NC, normal control. 


\section{Discussion}

Pregnancy-induced hypertension heart disease is the most common complication of HDCP, which is the leading cause of maternal and perinatal heart failure (16). The myocardial injury is a basic pathological change and manifests as apoptosis, inhibited proliferation, and necrosis, although the molecular mechanisms remain unclear (19). Studies indicate that miRNA serves an important role in myocardial injury (20). miR-150 was reported to protect myocardial ischemia by regulating apoptosis (21). Chen et al (22) revealed that overexpression of miR-214 increased myocardial cell injury through downregulating itchy E3 ubiquitin protein ligase target gene. In viral myocarditis, Bao and Lin (23) identified that miR-155 and miR148a protect myocardial cells through repressing the nuclear factor- $\mathrm{\kappa B}$-signaling pathway. The TIMP3 protein belongs to the TIMPs family, which serve as inhibitors for MMPs (24). TIMP3 is the only insoluble protein that strengthens the protection of the extracellular matrix (ECM), which participates in tumor metastasis, angiogenesis and other biological processes such as apoptosis and autophagy (25). TIMP3 was demonstrated to promote endothelial cell apoptosis by activating the Caspase signaling pathway (26). Zhou et al (27) indicated that miR-21 and miR-222 inhibited the apoptosis of neural cells by downregulating TIMP3. It was revealed that MMPs are secreted by trophoblastic cells, which are able to degrade the ECM as a restriction enzyme in the processes of trophoblastic cell invasion (28). The invasion degree of trophoblastic cells is associated with the ischemia of trophoblastic cells, which is the primary cause of HDCP (29). TIMP3 may combine with MMPs to sustain the balance of the degradation of ECM in physiological environment (30-32). The present study hypothesized that abnormally expressed TIMP3 in the placenta was associated with HDCP in pregnancy. However, there are few studies regarding the role of TIMP3 in myocardial injury.

As an important post-transcriptional regulator, miRNA is reported to participate the development and progression of HDCP (33). miRNA broadly exists in body fluid, such as blood, saliva and urine (34). The current study hypothesized that miRNA may be regarded as a biomarker. In the present study, the expression of miR-181b in peripheral blood of patients with HDCP was analyzed. miR-181b expression significantly decreased compared with normal puerpera and the expression was significantly lower in II/III stage than in stage I of hypertension $(\mathrm{P}<0.05)$. A sustained increase in blood pressure may damage the myocardial cells, which indicates that miR-181b may be associated with myocardial injury. To validate this hypothesis, the present study cultured primary rat cardiomyocytes in vitro and induced myocardial injury by hypoxia. As the time of hypoxia increased, miR-181b expression significantly decreased accordingly, while TIMP3 expression was significantly increased $(\mathrm{P}<0.05)$. Notably, the $\mathrm{miR}-181 \mathrm{~b}$ sequence in rats is consistent to the sequence of miR-181b in humans. Bioinformatic predictions indicated that TIMP3 was one of target genes of miR-181b and based on the dual luciferase reporter assay, miR-181b may regulate TIMP3 through directly binding to the 3'UTR of mRNA. miR-181b may serve its functions by directly targeting TIMP3. The current study further investigated the function of miR-181b through transfection with miR-181b mimics and inhibitors: Revealing that overexpressed miR-181b may inhibit myocardial cell apoptosis in a hypoxic environment and TIMP3 was downregulated accordingly. Following the downregulation of TIMP3 by siRNA transfection, the apoptosis rate of myocardial cells was significantly decreased in hypoxia conditions $(\mathrm{P}<0.05)$.

In conclusion, the results of the present study indicate that miR-181b may protect myocardial cells through targeting TIMP3 in heart disease induced by HDCP. The downregulation of miR-181b induces the increase of TIMP 3 expression, which promotes the apoptosis of myocardial cells and increases myocardial injury. However, these results were obtained in vitro, and further in vivo investigations are still required The results suggest that the MiR-181b monitor is of clinical significance in the diagnosis and management of myocardial injury.

\section{Acknowledgements}

The authors of the present study thank Professor Xiaohong Wang (Laiwu City People's Hospital) for help and support during research subject selection, experimental design, experiments, results analysis and paper preparation and modification.

\section{References}

1. Hofmeyr GJ, Lawrie TA, Atallah AN and Duley L: Calcium supplementation during pregnancy for preventing hypertensive disorders and related problems. Cochrane Database Syst Rev 4: CD001059, 2014.

2. Lin H, Yin Z, Yu XY, Lin N, Lin Y, Chen J, Chen YZ, Lu KP and Liu HK: Variants $-250 \mathrm{G} / \mathrm{A}$ and $-514 \mathrm{C} / \mathrm{T}$ in the LIPC gene are associated with hypertensive disorders of pregnancy in Chinese women. Genet Mol Res 13: 6126-6134, 2014.

3. Li LX, Liu YL, Wen JG, Li ZZ and Zhao YP: Expression and significance of aquaporin 1 in placenta, placental membranes and peritoneum of patients with hypertensive disorder complicating pregnancy. Zhonghua Fu Chan Ke Za Zhi 43: 497-501, 2008 (In Chinese).

4. Cairns AE, Tucker KL, Leeson P, Mackillop L and McManus RJ: Survey of healthcare professionals regarding adjustment of antihypertensive medication(s) in the postnatal period in women with hypertensive disorders of pregnancy. Pregnancy Hypertens 6: 256-258, 2016.

5. Zhong W, Peng H, Tian A, Wei Y, Li H, Tian J and Zhao X: Expression of miRNA-1233 in placenta from patients with hypertensive disorder complicating pregnancy and its role in disease pathogenesis. Int J Clin Exp Med 8: 9121-9127, 2015.

6. Gillon TE, Pels A, von Dadelszen P, MacDonell K and Magee LA: Hypertensive disorders of pregnancy: A systematic review of international clinical practice guidelines. PLoS One 9: e113715, 2014.

7. Bi GL, Chen FL and Huang WM: The association between hypertensive disorders in pregnancy and bronchopulmonary dysplasia: A systematic review. World J Pediatr 9: 300-306, 2013.

8. Schoenaker DA, Soedamah-Muthu SS, Callaway LK and Mishra GD: Prepregnancy dietary patterns and risk of developing hypertensive disorders of pregnancy: Results from the Australian Longitudinal Study on Women's Health. Am J Clin Nutr 102: 94-101, 2015.

9. Perveen S: Frequency and impact of hypertensive disorders of pregnancy. J Ayub Med Coll Abbottabad 26: 518-521, 2014.

10. Nishiguchi $T$, Imanishi $T$ and Akasaka T: MicroRNAs and cardiovascular diseases. Biomed Res Int 2015: 682857, 2015.

11. Pitari MR, Rossi M, Amodio N, Botta C, Morelli E, Federico C, Gullà A, Caracciolo D, Di Martino MT, Arbitrio M, et al: Inhibition of miR-21 restores RANKL/OPG ratio in multiple myeloma-derived bone marrow stromal cells and impairs the resorbing activity of mature osteoclasts. Oncotarget 6: 27343-27358, 2015. 
12. Wu XF, Zhou ZH and Zou J: MicroRNA-181 inhibits proliferation and promotes apoptosis of chondrocytes in osteoarthritis by targeting PTEN. Biochem Cell Biol: Jan 25, 2017 (Epub ahead of print).

13. Li D, Zhan S, Wang Y, Wang L, Zhong T, Li L, Fan J, Xiong C, Wang $\mathrm{Y}$ and Zhang $\mathrm{H}$ : Role of microRNA-101a in the regulation of goat skeletal muscle satellite cell proliferation and differentiation. Gene 572: 198-204, 2015.

14. Banzet S, Chennaoui M, Girard O, Racinais S, Drogou C, Chalabi $\mathrm{H}$ and Koulmann N: Changes in circulating microRNAs levels with exercise modality. J Appl Physiol (1985) 115: 1237-1244, 2013

15. Jiang X, Ning Q and Wang J: Angiotensin II induced differentially expressed microRNAs in adult rat cardiac fibroblasts. J Physiol Sci 63: 31-38, 2013.

16. Yuan T, Zhang T and Han Z: Placental vascularization alterations in hypertensive disorders complicating pregnancy (HDCP) and small for gestational age with HDCP using three-dimensional power doppler in a prospective case control study. BMC Pregnancy Childbirth 15: 240, 2015.

17. Monteiro MB, Santos-Bezerra DP, Thieme K, Passarelli M, Machado UF, Lin CJ and Corrêa-Giannella ML: Optimization of total RNA isolation from human urinary sediment. Clin Chim Acta 462: 158-161, 2016.

18. He J, Mai J, Li Y, Chen L, Xu H, Zhu X and Pan Q: miR-597 inhibits breast cancer cell proliferation, migration and invasion through FOSL2. Oncol Rep 37: 2672-2678, 2017.

19. Pang LZ, Ju AC, Zheng XJ, Li F, Song YF, Zhao Y, Gu YF, Chen FL, Liu CH, Qi J, et al: YiQiFuMai powder injection attenuates coronary artery ligation-induced myocardial remodeling and heart failure through modulating MAPKs signaling pathway. J Ethnopharmacol 202: 67-77, 2017.

20. Pandey R, Yang Y, Jackson L and Ahmed RP: MicroRNAs regulating meis1 expression and inducing cardiomyocyte proliferation. Cardiovasc Regen Med 3: pii: e1468, 2016.

21. Tang Y, Wang Y, Park KM, Hu Q, Teoh JP, Broskova Z, Ranganathan P, Jayakumar C, Li J, Su H, et al: MicroRNA-150 protects the mouse heart from ischaemic injury by regulating cell death. Cardiovasc Res 106: 387-397, 2015.

22. Chen ZG, Liu H, Zhang JB, Zhang SL, Zhao LH and Liang WQ: Upregulated microRNA-214 enhances cardiac injury by targeting ITCH during coxsackievirus infection. Mol Med Rep 12: 1258-1264, 2015

23. Bao JL and Lin L: MiR-155 and miR-148a reduce cardiac injury by inhibiting NF- $\kappa \mathrm{B}$ pathway during acute viral myocarditis. Eur Rev Med Pharmacol Sci 18: 2349-2356, 2014.
24. Meunier I, Bocquet B,Labesse G,Zeitz C, Defoort-Dhellemmes S, Lacroux A, Mauget-Faysse M, Drumare I, Gamez AS, Mathieu C, et al: A new autosomal dominant eye and lung syndrome linked to mutations in TIMP3 gene. Sci Rep 6: 32544 , 2016.

25. Petrovic N, Davidovic R, Jovanovic-Cupic S, Krajnovic M, Lukic S, Petrovic M and Roganovic J: Changes in miR-221/222 levels in invasive and in situ carcinomas of the breast: Differences in association with estrogen receptor and TIMP3 expression levels. Mol Diagn Ther 20: 603-615, 2016.

26. Qi JH and Anand-Apte B: Tissue inhibitor of metalloproteinase-3 (TIMP3) promotes endothelial apoptosis via a caspase-independent mechanism. Apoptosis 20: 523-534, 2015.

27. Zhou S, Zhang S, Wang Y, Yi S, Zhao L, Tang X, Yu B, Gu X and Ding F: MiR-21 and miR-222 inhibit apoptosis of adult dorsal root ganglion neurons by repressing TIMP3 following sciatic nerve injury. Neurosci Lett 586: 43-49, 2015.

28. Sun LZ, Ma XT, Ge ZP and Han P: Effect of endoplasmic reticulum stress-responsive protein glucose-regulated protein 78 , 94 and endoplasmic reticulum apoptosis factor caspase-12 in trophocyte on the pathogenesis of preeclampsia. Zhonghua $\mathrm{Fu}$ Chan Ke Za Zhi 45: 891-895, 2010 (In Chinese).

29. Shah DA and Khalil RA: Bioactive factors in uteroplacental and systemic circulation link placental ischemia to generalized vascular dysfunction in hypertensive pregnancy and preeclampsia. Biochem Pharmacol 95: 211-226, 2015.

30. Sternlicht MD and Werb Z: How matrix metalloproteinases regulate cell behavior. Annu Rev Cell Dev Biol 17: 463-516, 2001.

31. Poulet B, Liu K, Plumb D, Vo P, Shah M, Staines K, Sampson A, Nakamura H, Nagase H, Carriero A, et al: Overexpression of TIMP-3 in chondrocytes produces transient reduction in growth plate length but permanently reduces adult bone quality and quantity. PLoS One 11: e0167971, 2016.

32. Fu X, Zeng L, Liu Z, Ke X, Lei L and Li G: MicroRNA-206 regulates the secretion of inflammatory cytokines and MMPO expression by targeting TIMP3 in Mycobacterium tuberculosis-infected THP-1 human macrophages. Biochem Biophys Res Commun 477: 167-173, 2016.

33. Wu L, Xiong C and Su P: Endogenous ouabain in hypertensive disorder complicating pregnancy. J Huazhong Univ Sci Technolog Med Sci 27: 717-720, 2007.

34. Zhang Y, Huang B, Wang HY, Chang A and Zheng XF: Emerging role of MicroRNAs in mTOR signaling. Cell Mol Life Sci: Feb 25, 2017 (Epub ahead of print). 